\title{
Two-Step Simulations of Reaction Systems by Minimal Ones
}

\author{
Arto Salomaa*
}

\begin{abstract}
Reaction systems were introduced by Ehrenfeucht and Rozenberg with biochemical applications in mind. The model is suitable for the study of subset functions, that is, functions from the set of all subsets of a finite set into itself. In this study the number of resources of a reaction system is essential for questions concerning generative capacity. While all functions (with a couple of trivial exceptions) from the set of subsets of a finite set $S$ into itself can be defined if the number of resources is unrestricted, only a specific subclass of such functions is defined by minimal reaction systems, that is, the number of resources is smallest possible. On the other hand, minimal reaction systems constitute a very elegant model. In this paper we simulate arbitrary reaction systems by minimal ones in two derivation steps. Various techniques for doing this consist of taking names of reactions or names of subsets as elements of the background set. In this way also subset functions not at all definable by reaction systems can be generated. We follow the original definition of reaction systems, where both reactant and inhibitor sets are assumed to be nonempty
\end{abstract}

Keywords: reaction system, reactant, inhibitor, minimal resources, subset function, sequence

\section{Introduction}

A formal model of reaction systems was introduced by Ehrenfeucht and Rozenberg in [3]. Everything is defined within a fixed finite background set $S$. The original purpose was to model interactions between biochemical reactions. The reference [3] contains some of the original motivation and initial setup. Each reaction is characterized by its set of reactants, each of which has to be present for the reaction to take place, by its set of inhibitors, none of which is allowed to be present, and by its set of products, each of which will be present after a successful reaction. Thus, a single reaction is based on facilitation and inhibition.

\footnotetext{
*Turku Centre for Computer Science. Joukahaisenkatu 3-5 B, 20520 Turku, Finland. E-mail:
} asalomaa@utu.fi 
Reaction systems provide a new kind of mechanism for generating functions and sequences over a finite set. A reaction system produces (in a way explained below) another subset $Y$ of $S$ and, thus, we have a subset function from subsets of $S$ to subsets of $S$. Iterating the function we get a sequence of subsets of $S$. The theory of subset functions has been studied from many points of view, [15], and is particularly important in many-valued logic, [8].

Many variants of reaction systems have been introduced. The reference [1] constitutes a survey. However, the very active research in this area opens frequently new vistas. We refer to [4] for quite new developments.

Apart from various applications, reaction systems as such have been objected to many theoretical studies, $[2,5,9,10,11,12,13]$. The arising problems are mathematically very interesting, since the model is simple and clean.

However, in this paper we are concerned with the basic variant only and follow the original definition.

The elements of the sets of reactants and inhibitors are also referred to as resources of the reaction. Since both sets are by definition nonempty and disjoint, the smallest possible cardinality of the resource set equals 2. Such minimal reaction systems constitute a very simple and interesting model of computation. The class of subset functions defined by minimal reaction systems was characterized in [2]. As to be expected, the class is much smaller than the one defined by arbitrary reaction systems.

In this paper we try to narrow the gap between the generative capacities of arbitrary and minimal reaction systems. The method used below is a two-step simulation. Starting with an arbitrary reaction system, we construct a minimal one such that an arbitrary sequence of the former can be read from a sequence of the latter by taking every second subset: first, third, fifth,... The remaining subsets (second, fourth,...) contain only "junk" elements outside the background set of the original reaction system.

The exposition in this paper is largely self-contained. In particular, the basic definitions concerning reaction systems are given in Section 2 . We define only the core apparatus, and do not enter additions such as a sequence of inputs from the environment, $[3,1]$.

\section{Definitions and earlier results}

We begin by defining the basic notions.

Definition 1. A reaction over the finite nonempty background set $S$ is a triple

$$
\rho=(R, I, P)
$$

where $R, I$ and $P$ are nonempty subsets of $S$ such that $R$ and $I$ do not intersect. The three sets are referred as reactants, inhibitors and products, respectively. $A$ reaction system $\mathcal{A}_{S}$ over the background set $S$ is a finite nonempty set

$$
\mathcal{A}_{S}=\left\{\rho_{j} \mid 1 \leq j \leq k\right\}
$$


of reactions over $S$.

In this paper $S$ will always denote the background set. It is nonempty and finite. By subset functions we mean functions mapping the set $2^{S}$ into itself.

We will follow the original definition in [3] (motivated by biochemical considerations) and assume that both of the sets $R$ and $I$ are nonempty. It is also of definite interest to develop the theory without this assumption. This gives rise to many interesting constructions, also concerning stepwise simulation, see [6].

We will omit the index $S$ from $\mathcal{A}_{S}$ whenever $S$ is understood. The cardinality of a finite set $X$ is denoted by $\sharp X$. The empty set is denoted by $\emptyset$. We now indicate how reactions and reaction systems are used to define subset functions.

Definition 2. Consider a reaction $\rho=(R, I, P)$ over $S$ and a subset $T$ of $S$. The reaction $\rho$ is enabled with respect to $T$ (or for $T$ ), in symbols en $\rho(T)$, if $R \subseteq T$ and $I \cap T=\emptyset$. If $\rho$ is (resp. is not) enabled, then we define the result by

$$
\operatorname{res}_{\rho}(T)=P(\text { resp. }=\emptyset) .
$$

For a reaction system $\mathcal{A}=\left\{\rho_{j} \mid 1 \leq j \leq k\right\}$, we define the result by

$$
\operatorname{res}_{\mathcal{A}}(T)=\bigcup_{j=1}^{k} \operatorname{res}_{\rho_{j}}(T) .
$$

An important fact to notice is that, according to Definition 2, an element in the set $T$ is not "consumed" in the application of a reaction but is also available for other reactions when $\operatorname{res}_{\mathcal{A}}(T)$ is computed. In the sequel we often refer to $r e s_{\mathcal{A}}$ as the function defined by the reaction system $\mathcal{A}$.

Elements in the set $R \cup I$ are also referred to as resources. Reaction systems are classified according to the maximal cardinality of the set of resources. We have $\sharp(R \cup I) \geq 2$, since the sets $R$ and $I$ are nonempty and disjoint. A reaction system is minimal if $\sharp(R \cup I)=2$ holds for every reaction in the system. There is much research concerning minimal reaction systems, for instance, see $[2,7,9,10,12$, $13,14]$. The capacity of minimal reaction systems for defining subset functions is limited. We now quote the following fundamental result from [2], where the capacity is characterized.

Definition 3. A subset function $f$ is

- union-subadditive if $f(X \cup Y) \subseteq f(X) \cup f(Y)$,

- intersection-subadditive if $f(X \cap Y) \subseteq f(X) \cup f(Y)$,

for all subsets $X$ and $Y$ of $S$.

The characterization result in [2] is now given

Theorem 1. A function defined by a reaction system is definable by a minimal reaction system if and only if it is both union-subadditive and intersection-subadditive. 
Sequences generated by reaction systems can be viewed as iterations of functions $r e s_{\mathcal{A}}$. If $\operatorname{res}_{\mathcal{A}}(T)=T^{\prime}$, we use the simple notation

$$
T \Rightarrow_{\mathcal{A}} T^{\prime}
$$

or simply $T \Rightarrow T^{\prime}$ if $\mathcal{A}$ is understood. If

$$
\operatorname{res}_{\mathcal{A}}\left(T_{i}\right)=T_{i+1}, 0 \leq i \leq m-1,
$$

we write briefly

$$
T_{0} \Rightarrow T_{1} \Rightarrow \ldots \Rightarrow T_{m}
$$

and call $m$ the length of the sequence. Since there are only $2^{\sharp S}$ subsets of $S$, there is always an $m$ such that, for some $m_{1}<m, T_{m}=T_{m_{1}}$, or else $\operatorname{res}_{\mathcal{A}}\left(T_{m-1}\right)$ is undefined, in which case we write $T_{m}=\emptyset$. We say that the sequence ends with a cycle or terminates, respectively. The sets $T_{i}$ are usually referred to as states of the sequence.

Maximally inhibited reaction systems, [9, 14], offer possibilities of constructing arbitrary sequences or cycles.

Definition 4. A reaction system with the background set $S$ is maximally inhibited if every one of its reactions is of the form $(R, S-R, P)$.

Clearly, for every reaction system $\mathcal{A}$, a maximally inhibited reaction system $\mathcal{A}^{\prime}$ can be constructed such that, for any $T$,

$$
\operatorname{res}_{\mathcal{A}}(T)=\operatorname{res}_{\mathcal{A}^{\prime}}(T) .
$$

If $\operatorname{res}_{\mathcal{A}}(T)=\emptyset$, then there is no reaction in $\mathcal{A}^{\prime}$, where $T$ is the set of reactants.

\section{Names of reactions as elements of the back- ground set}

We now present our main result concerning the two-step simulation of arbitrary reaction systems by minimal ones. We have earlier, [14], presented another form of a similar construction. Names of reactions have been used as elements of the background set also in [5]. Our result shows that if one starts with a sequence (or cycle)

$$
T_{0} \Rightarrow T_{1} \Rightarrow \ldots \Rightarrow T_{m} \ldots
$$

according to an arbitrary reaction system $\mathcal{A}_{S}$, then a minimal reaction system $\mathcal{A}_{M}$ with the sequence

$$
T_{0} \Rightarrow U_{0} \Rightarrow \ldots T_{1} \Rightarrow U_{1} \Rightarrow T_{2} \ldots
$$

can be constructed. The background set $S_{M}$ of $\mathcal{A}_{M}$ includes $S$. Moreover, the intermediate states $U_{i}$ contain only elements of $S_{M}-S$ and, thus, are analogous to nonterminals in grammars. 
Theorem 2. For every reaction system $\mathcal{A}$, a minimal reaction system $\mathcal{A}_{M}$ can be effectively constructed such that, whenever $T_{0} \Rightarrow_{\mathcal{A}} T_{1}$, then $T_{0} \Rightarrow_{\mathcal{A}_{M}} U_{0} \Rightarrow_{\mathcal{A}_{M}} T_{1}$. Moreover, the set $U_{0}$ does not contain elements of the background set of $\mathcal{A}$.

Proof. Let $\mathcal{A}$ have the background set $S$ and the set of reactions

$$
\rho_{i}=\left(R_{i}, I_{i}, P_{i}\right), 1 \leq i \leq k .
$$

We now define the minimal reaction system $\mathcal{A}_{M}$. Its background set is

$$
S_{M}=S \cup\left\{\rho_{1}, \ldots \rho_{k}, E\right\} .
$$

It will be convenient to divide its reactions into three groups.

The first group consists of taking, for every $a \in S$, the reaction

$$
\left(\{a\},\{E\},\left\{\rho_{i_{1}}, \ldots, \rho_{i_{m}}, E\right\}, a \in I_{i_{j}}, 1 \leq j \leq m .\right.
$$

No reaction results if $a$ does not belong to any inhibitor set.

The second group consists of taking, for every $a \in S$, the reactions

$$
\left(\{b\},\{a\},\left\{\rho_{j_{1}}, \ldots, \rho_{j_{n}}, E\right\}, a \in R_{j_{\nu}}, 1 \leq \nu \leq n, b \in S, b \neq a .\right.
$$

No reaction results if $a$ does not belong to any reactant set.

The third group consists of reactions

$$
\left(\{E\},\left\{\rho_{i}\right\}, P_{i}\right), 1 \leq i \leq k .
$$

If a sequence of $\mathcal{A}_{M}$ begins with $\emptyset$, there is nothing to prove. Thus, consider a nonempty $T \subseteq S$. We claim that the second state in the sequence beginning with $T$ consists of $E$ and the names of those reactions $\rho_{i}$ for which $e n_{\rho_{i}}(T)$ does NOT hold. Then only some reactions $\left(\{E\},\left\{\rho_{i}\right\}, P_{i}\right)$ of the third group are enabled, namely, exactly those for which $e n_{\rho_{i}}(T)$ holds. Consequently, the third state in the sequence equals $\operatorname{res}_{\mathcal{A}_{M}}(T)$, and Theorem 2 follows.

To prove our claim, note first that $E$ is always present in the second state, whereas no elements of $S$ are present. We have to show that, whenever $e n_{\rho_{i}}(T)$ does not hold, then $\rho_{i}$ is present in the second state. By Definition 2, the relation $e n_{\rho_{i}}(T)$ does not hold if and only if either

1. $a_{1} \in T \cap I_{i}$, for some $a_{1}$, or else,

2. $a_{2} \in R_{i}-T$, for some $a_{2}$.

Reactions in (1) (resp. in (2)) appear in the product set of the first (resp. the second) group of reactions of $\mathcal{A}_{M}$. Consequently, exactly those reactions $\rho_{i}$ from the set $\left\{\rho_{1}, \ldots, \rho_{k}\right\}$ are missing from the second state of the sequence for which $e n_{\rho_{i}}(T)$ holds.

As an example consider the reaction system $\mathcal{A}$ with the background set $S=$ $\{a, b, c\}$ and reactions

$$
\begin{array}{r}
\rho_{1}=(\{a, c\},\{b\},\{b\}), \rho_{2}=(\{b, c\},\{a\},\{a, b\}), \\
\rho_{3}=(\{b\},\{a, c\},\{a, b, c\}), \rho_{4}=(\{c\},\{a, b\},\{a, c\}) .
\end{array}
$$


Now the minimal reaction system $\mathcal{A}_{M}$ has the background set

$$
\left\{a, b, c, \rho_{1}, \rho_{2}, \rho_{3}, \rho_{4}, E\right\}
$$

and reactions

$$
\begin{array}{r}
\left(\{a\},\{E\},\left\{\rho_{2}, \rho_{3}, \rho_{4}, E\right\}\right),\left(\{b\},\{E\},\left\{\rho_{1}, \rho_{4}, E\right\}\right),\left(\{c\},\{E\},\left\{\rho_{3}, E\right\}\right), \\
\left(\{b\},\{a\},\left\{\rho_{1}, E\right\}\right),\left(\{c\},\{a\},\left\{\rho_{1}, E\right\}\right),\left(\{a\},\{b\},\left\{\rho_{2}, \rho_{3}, E\right\}\right),
\end{array}
$$

$\left(\{c\},\{b\},\left\{\rho_{2}, \rho_{3}, E\right\}\right),\left(\{a\},\{c\},\left\{\rho_{1}, \rho_{2}, \rho_{4}, E\right\}\right),\left(\{b\},\{c\},\left\{\rho_{1}, \rho_{2}, \rho_{4}, E\right\}\right)$, $\left(\{E\},\left\{\rho_{1}\right\},\{b\}\right),\left(\{E\},\left\{\rho_{2}\right\},\{a, b\}\right),\left(\{E\},\left\{\rho_{3}\right\},\{a, b, c\}\right),\left(\{E\},\left\{\rho_{4}\right\},\{a, c\}\right)$

The first two steps in the sequence of $\mathcal{A}_{M}$ are listed below, beginning with the 6 possible nonempty proper subsets of $S$.

$$
\begin{array}{r}
\{a, b\} \Rightarrow\left\{\rho_{1}, \rho_{2}, \rho_{3}, \rho_{4}, E\right\} \Rightarrow \emptyset \\
\{a, c\} \Rightarrow\left\{\rho_{2}, \rho_{3}, \rho_{4}, E\right\} \Rightarrow\{b\} \\
\{b, c\} \Rightarrow\left\{\rho_{1}, \rho_{3}, \rho_{4}, E\right\} \Rightarrow\{a, b\} \\
\{a\} \Rightarrow\left\{\rho_{1}, \rho_{2}, \rho_{3}, \rho_{4}, E\right\} \Rightarrow \emptyset \\
\{b\} \Rightarrow\left\{\rho_{1}, \rho_{2}, \rho_{4}, E\right\} \Rightarrow\{a, b, c\} \\
\{c\} \Rightarrow\left\{\rho_{1}, \rho_{2}, \rho_{3}, E\right\} \Rightarrow\{a, c\}
\end{array}
$$

Our reaction system $\mathcal{A}$ is maximally inhibited and, consequently, each of the values $\operatorname{res}_{\mathcal{A}}(T)$ can be seen directly from the reaction, where $T$ is the set of reactants. This is not the case with our second example, where the reaction system is not maximally inhibited.

Thus, consider now consider the reaction system $\mathcal{A}$ with the background set $S=\{a, b, c, d\}$ and reactions

$$
\begin{array}{r}
\rho_{1}=(\{a, b\},\{c\},\{b, d\}), \rho_{2}=(\{a\},\{b, c\},\{a\}), \\
\rho_{3}=(\{a\},\{c, d\},\{c\}), \rho_{4}=(\{b, c\},\{a, d\},\{a, c\}), \\
\rho_{5}=(\{c\},\{a, b\},\{a, b, c, d\}), \rho_{6}=(\{a, d\},\{c\},\{a, b, c\}) .
\end{array}
$$

By the construction of Theorem 2, the minimal reaction system $\mathcal{A}_{M}$ has the background set

$$
\left\{a, b, c, d, \rho_{1}, \rho_{2}, \rho_{3}, \rho_{4}, \rho_{5}, \rho_{6}, E\right\}
$$

and reactions

$$
\left(\{a\},\{E\},\left\{\rho_{4}, \rho_{5}, E\right\}\right),\left(\{b\},\{E\},\left\{\rho_{2}, \rho_{5}, E\right\}\right),\left(\{c\},\{E\},\left\{\rho_{1}, \rho_{2}, \rho_{3}, \rho_{6}, E\right\}\right),
$$
$\left(\{d\},\{E\},\left\{\rho_{3}, \rho_{4}, E\right\}\right),\left(\{b\},\{a\},\left\{\rho_{1}, \rho_{2}, \rho_{3}, \rho_{6}, E\right\}\right),\left(\{c\},\{a\},\left\{\rho_{1}, \rho_{2}, \rho_{3}, \rho_{6}, E\right\}\right)$,

$\left(\{d\},\{a\},\left\{\rho_{1}, \rho_{2}, \rho_{3}, \rho_{6}, E\right\}\right),\left(\{a\},\{b\},\left\{\rho_{1}, \rho_{4}, E\right\}\right),\left(\{c\},\{b\},\left\{\rho_{1}, \rho_{4}, E\right\}\right)$, $\left(\{d\},\{b\},\left\{\rho_{1}, \rho_{4}, E\right\}\right),\left(\{a\},\{c\},\left\{\rho_{4}, \rho_{5}, E\right\}\right),\left(\{b\},\{c\},\left\{\rho_{4}, \rho_{5}, E\right\}\right)$, $\left(\{d\},\{c\},\left\{\rho_{4}, \rho_{5}, E\right\}\right),\left(\{a\},\{d\},\left\{\rho_{6}, E\right\}\right),\left(\{b\},\{d\},\left\{\rho_{6}, E\right\}\right)$, $\left(\{c\},\{d\},\left\{\rho_{6}, E\right\}\right),\left(\{E\},\left\{\rho_{1}\right\},\{b, d\}\right),\left(\{E\},\left\{\rho_{2}\right\},\{a\}\right),\left(\{E\},\left\{\rho_{3}\right\},\{c\}\right)$, $\left(\{E\},\left\{\rho_{4}\right\},\{a, c\},\left(\{E\},\left\{\rho_{5}\right\},\{a, b, c, d\}\right),\left(\{E\},\left\{\rho_{6}\right\},\{a, b, c\}\right)\right.$. 
The two-step simulation by $\mathcal{A}_{M}$ of the original reaction system $\mathcal{A}$ is exhibited in the following exhaustive list.

$$
\begin{array}{r}
\{a\} \Rightarrow\left\{\rho_{1}, \rho_{4}, \rho_{5}, \rho_{6}, E\right\} \Rightarrow\{a, c\}, \\
\{b\} \Rightarrow\left\{\rho_{1}, \rho_{2}, \rho_{3}, \rho_{4}, \rho_{5}, \rho_{6}, E\right\} \Rightarrow \emptyset, \\
\{c\} \Rightarrow\left\{\rho_{1}, \rho_{2}, \rho_{3}, \rho_{4}, \rho_{6}, E\right\} \Rightarrow\{a, b, c, d\}, \\
\{d\} \Rightarrow\left\{\rho_{1}, \rho_{2}, \rho_{3}, \rho_{4}, \rho_{5}, \rho_{6}, E\right\} \Rightarrow \emptyset, \\
\{a, b\} \Rightarrow\left\{\rho_{2}, \rho_{4}, \rho_{5}, \rho_{6}, E\right\} \Rightarrow\{b, c, d\}, \\
\{a, c\} \Rightarrow\left\{\rho_{1}, \rho_{2}, \rho_{3}, \rho_{4}, \rho_{5}, \rho_{6}, E\right\} \Rightarrow \emptyset, \\
\{a, d\} \Rightarrow\left\{\rho_{1}, \rho_{3}, \rho_{4}, \rho_{5}, E\right\} \Rightarrow\{a, b, c\}, \\
\{b, c\} \Rightarrow\left\{\rho_{1}, \rho_{2}, \rho_{3}, \rho_{5}, \rho_{6}, E\right\} \Rightarrow\{a, c\}, \\
\{b, d\} \Rightarrow\left\{\rho_{1}, \rho_{2}, \rho_{3}, \rho_{4}, \rho_{5}, \rho_{6}, E\right\} \Rightarrow \emptyset, \\
\{c, d\} \Rightarrow\left\{\rho_{1}, \rho_{2}, \rho_{3}, \rho_{4}, \rho_{6}, E\right\} \Rightarrow\{a, b, c, d\}, \\
\{a, b, c\} \Rightarrow\left\{\rho_{1}, \rho_{2}, \rho_{3}, \rho_{4}, \rho_{5}, \rho_{6}, E\right\} \Rightarrow \emptyset, \\
\{a, b, d\} \Rightarrow\left\{\rho_{2}, \rho_{3}, \rho_{4}, \rho_{5}, E\right\} \Rightarrow\{a, b, c, d\}, \\
\{a, c, d\} \Rightarrow\left\{\rho_{1}, \rho_{2}, \rho_{3}, \rho_{4}, \rho_{5}, \rho_{6}, E\right\} \Rightarrow \emptyset, \\
\{b, c, d\} \Rightarrow\left\{\rho_{1}, \rho_{2}, \rho_{3}, \rho_{4}, \rho_{5}, \rho_{6}, E\right\} \Rightarrow \emptyset .
\end{array}
$$

The following result follows directly from the proof of Theorem 2 .

Corollary 1. Assume that the background set and the set of reactions of a arbitrary reaction system $\mathcal{A}$ are of cardinalities $s$ and $k$, respectively. Then a minimal reaction system $\mathcal{A}_{M}$ satisfying Theorem 2 can be effectively constructed such that the cardinalities of its background and reaction sets are $s+k+1$ and $s^{2}+k$, respectively.

Corollary 1 is pleasing because it allows the extension of some results concerning computational complexity of reaction systems to minimal reaction systems. We hope to return to these matters in another context.

\section{Extension to subset functions}

Reaction systems provide a new formal tool of handling subset functions, that is, functions from $2^{S}$ into $2^{S}$, where $S$ is a finite set. This is an important aspect of reaction systems.

We begin with the following result.

Corollary 2. Let $F(X)$ be a function mapping the set of all nonempty proper subsets of a finite set $S$ into the set of all subsets of $S$. Then there is (effectively) a minimal reaction system $\mathcal{A}_{M}$ such that

$$
F(X)=\operatorname{res}_{\mathcal{A}_{M}}^{2}(X) .
$$


Proof. The claim follows by Theorem 2 by starting with the maximally inhibited reaction system $\mathcal{A}$ with reactions $(X, S-X, F(X))$, where $X$ runs through all nonempty proper subsets of the background set. If $F(X)$ is empty, the corresponding triple is not among the reactions of $\mathcal{A}_{M}$.

It is not possible to extend Corollary 2 to concern functions $F$ with $F(\emptyset) \neq \emptyset$. No reaction can produce anything nonempty from the empty set. We will now prove that this is, in fact, the only exception.

Theorem 3. Let $F$ be a subset function over the set $S$ such that $F(\emptyset)=\emptyset$. There is effectively a minimal reaction system $\mathcal{A}_{M}$ such that, for all $X \subseteq S$,

$$
F(X)=\operatorname{res}_{\mathcal{A}_{M}}^{2}(X) .
$$

Proof. We follow the proof of Theorem 2. We have to take care of the case, where the whole background set $S$ appears as an argument of the function $F$. Assume first that $F(S)=\emptyset$. Then the proof of Theorem 2 works without any changes. The second state of the sequence of $\mathcal{A}_{M}$, starting with $S$, is $\left\{E, \rho_{1}, \ldots, \rho_{k}\right\}$. Thus, none of the reactions of the third group is enabled, yielding $\emptyset$.

Assume, secondly, that $F(S) \neq \emptyset$. In this case we make the following additions to the reaction system $\mathcal{A}_{M}$. The element $\rho_{S}$ is added to the background set of $\mathcal{A}_{M}$. For every element $a \in S$, the element $\rho_{S}$ is added to the product set of each reaction in the second group. The reaction $\left(\{E\},\left\{\rho_{S}\right\}, F(S)\right)$ is added to the third group. It is now easy to verify that

$$
F(X)=\operatorname{res}_{\mathcal{A}_{M}}^{2}(X)
$$

holds for all $X \subseteq S$.

As an example we consider the background set $S=\{a, b, c\}$ and the subset function $F$ defined by

\begin{tabular}{c|cccccccc}
$X$ & $\emptyset$ & $\{a\}$ & $\{b\}$ & $\{c\}$ & $\{a, b\}$ & $\{a, c\}$ & $\{b, c\}$ & $\{a, b, c\}$ \\
\hline$F(X)$ & $\emptyset$ & $\emptyset$ & $\{a, b, c\}$ & $\{a, c\}$ & $\emptyset$ & $\{b\}$ & $\{a, b\}$ & $\{a, b\}$
\end{tabular}

This example is a modification of the first example in the preceding section.

We now define the minimal reaction system $\mathcal{A}_{M}$ as in Theorem 3. The minimal reaction system $\mathcal{A}_{M}$ has the background set

$$
\left\{a, b, c, \rho_{1}, \rho_{2}, \rho_{3}, \rho_{4}, \rho_{S}, E\right\}
$$

and reactions

$$
\begin{array}{r}
\left(\{a\},\{E\},\left\{\rho_{2}, \rho_{3}, \rho_{4}, E\right\}\right),\left(\{b\},\{E\},\left\{\rho_{1}, \rho_{4}, E\right\}\right),\left(\{c\},\{E\},\left\{\rho_{3}, E\right\}\right), \\
\left(\{b\},\{a\},\left\{\rho_{1}, \rho_{S}, E\right\}\right),\left(\{c\},\{a\},\left\{\rho_{1}, \rho_{S}, E\right\}\right),\left(\{a\},\{b\},\left\{\rho_{2}, \rho_{3}, \rho_{S}, E\right\}\right), \\
\left(\{c\},\{b\},\left\{\rho_{2}, \rho_{3}, \rho_{S}, E\right\}\right),\left(\{a\},\{c\},\left\{\rho_{1}, \rho_{2}, \rho_{4}, \rho_{S}, E\right\}\right), \\
\left(\{b\},\{c\},\left\{\rho_{1}, \rho_{2}, \rho_{4}, \rho_{S}, E\right\}\right),\left(\{E\},\left\{\rho_{1}\right\},\{b\}\right),\left(\{E\},\left\{\rho_{2}\right\},\{a, b\}\right), \\
\left(\{E\},\left\{\rho_{3}\right\},\{a, b, c\}\right),\left(\{E\},\left\{\rho_{4}\right\},\{a, c\}\right),\left(\{E\},\left\{\rho_{S}\right\},\{a, b\}\right) .
\end{array}
$$


We obtain now

$$
\{a, b, c\} \Rightarrow\left\{\rho_{1}, \rho_{2}, \rho_{3}, \rho_{4}, E\right\} \Rightarrow\{a, b\}
$$

It is easy to see that, for any $X \subseteq S$, the function value $F(X)$ appears as the third state in the sequence beginning with $X$.

\section{Names of subsets as elements of the background set}

We present for the sake of completeness the following result due to [7]. It uses names of subsets, rather than names of reactions, as an extension of the background set. While this approach is mathematically elegant, it leads to huge background sets. Apparently it can also not be extended to subset functions as Theorem 3.

Theorem 4. For a maximally inhibited reaction system $\mathcal{A}$ with the background set $S$, there is effectively a minimal reaction system $\mathcal{A}_{M}$ such that, for every proper subset $X$ of $S$,

$$
\operatorname{res}_{\mathcal{A}}(X)=\operatorname{res}_{\mathcal{A}_{M}}^{2}(X)
$$

Proof. Since $\mathcal{A}$ is maximally inhibited, to test whether or not $e n_{\rho}(X)$ for $\rho=$ $(R, I, P)$, it suffices to test whether or not $X=R$. The proof is based on this observation.

We introduce a new symbol $N_{X}$ (name of $X$ ), for every subset $X$ of $S$. The background set of $\mathcal{A}_{M}$ is now $S \cup\left\{N_{X} \mid X \subseteq S\right\}$. Now we use the fact that, for any $X$, at most one reaction in $\mathcal{A}$ is enabled with respect to $X$, namely, the reaction $\left(X, S-X, P_{X}\right)$. As observed, to check whether or not not a reaction is enabled with respect to the first state $X$ in a sequence, we only have to eliminate reactions whose reactant set is not $X$. This can be accomplished using the first group of reactions in $\mathcal{A}_{M}$

$$
\left(\{a\},\{b\},\left\{N_{X}\right\}\right), X \subseteq S, a \in S-X, \text { or } b \in X, a \neq b
$$

These reactions produce the name $N_{Y}$ if and only if $Y \neq X$. This means that only the correct $N_{X}$ is missing from the second state of the sequence.

The second group of reactions in $\mathcal{A}_{M}$ consists of the following reactions

$$
\left(\left\{N_{Y}\right\},\left\{N_{X}\right\}, P_{X}\right), X, Y \subseteq S, X \neq Y, X \neq S, \emptyset
$$

These reactions yield the set $P_{X}$ as the third state.

Considering sequences, every second state consists of "junk" elements and is of size $2^{s}-1$, where $s$ is the cardinality of $S$. 


\section{Conclusion}

Functions defined by minimal reaction system were characterized in [2]. However, the conditions of the characterization are very hard to test. Therefore, methods of transition from arbitrary reaction systems to minimal ones should be investigated. In this paper we have investigated the method of stepwise simulation. It is conceivable that better results are obtained by a different choice of objects whose names are used in the construction. It is a general open problem to investigate the gap between the class of functions defined by minimal and almost minimal reaction systems ( where the cardinality of the resource set is at most 3 in every reaction). We conjecture that this gap is bigger than the corresponding gap between almost minimal and general reaction systems.

\section{References}

[1] R. Brijder, A. Ehrenfeucht, M. Main and G. Rozenberg, A tour of reaction systems. International Journal of Foundations of Computer Science 22 (2011) 1499-1517.

[2] A. Ehrenfeucht, J.Kleijn, M. Koutny and G. Rozenberg, Minimal reaction systems. Springer Lecture Notes in Computer Science, Vol. 7625 (2012) 102122.

[3] A. Ehrenfeucht and G. Rozenberg, Reaction systems. Fundamenta Informaticae 7 (2007) 263-280.

[4] A. Ehrenfeucht and G. Rozenberg, Zoom structures and reaction systems yield exploration systems. International Journal of Foundations of Computer Science, 25 (2014) 275-305.

[5] E. Formenti, L. Manzoni and A.E. Porreca, On the complexity of occurrence and convergence problems for reaction systems. Natural Computing, DOI 10.1007/s11047-014-9456-3.

[6] L. Manzoni, D. Poças and A.E. Porreca, Simple reaction systems and their classification. International Journal of Foundations of Computer Science 25 (2014) 441-457.

[7] F. Montagna and G. Rozenberg, On minimal reaction systems, forthcoming. (G. Rozenberg, personal communication.)

[8] A. Salomaa, Composition sequences of functions over a finite domain. Theoretical Computer Science 292 (2003) 263-281.

[9] A. Salomaa, On state sequences defined by reaction systems. Springer Lecture Notes in Computer Science, Vol. 7230 (2012) 271-282. 
[10] A. Salomaa, Functions and sequences generated by reaction systems. Theoretical Computer Science 466 (2012) 87-96.

[11] A. Salomaa, Functional constructions between reaction systems and propositional logic. International Journal of Foundations of Computer Science 24 (2013) 147-159.

[12] A. Salomaa, Minimal and almost minimal reaction systems. Natural Computing 12 (2013) 369-376.

[13] A. Salomaa, Compositions of reaction systems. Journal of Automata, Languages and Combinatorics 19 (2014) 279-290.

[14] A. Salomaa, Minimal reaction systems defining subset functions, Springer Lecture Notes in Computer Science 8808 (2014) 436-446.

[15] P. Zhu, h.Xie and Q. Wen, Unified definition of consistent functions. Fundamenta Informaticae 135 (2014) 331-340.

\section{Dedication}

This paper is dedicated to the memory of Ferenc Gécseg, a great scientist and a close personal friend of mine. Although the topic of the paper is not related to his work, I still consider the topic appropriate because Ferenc was always interested in new ideas and notions.

I met Ferenc in a conference arranged by him in Szeged in August 1973. We immediately shared common interests in automata theory. Besides, he was very friendly and helped with my wife's health problem as well as with our minor car accident. This was the beginning of a vigorous scientific cooperation and close friendship that extended to concern also our families. Eventually we started to call each other Super-Brothers. Our children met frequently, and so did some of our grandchildren. However, one cannot have everything. Our planned family meeting, with all children and grandchildren, never materialized.

Ferenc and his family spent in Turku the academic year 1974-75. The scientific cooperation, started then, continued for decades and extended to concern many of our students and colleagues. It took the form of joint papers and books, mutual visits, and conferences jointly organized. Ferenc spent several longer periods in Turku and I paid some twenty visits to Szeged. Ferenc became a member of Finland's Academy of Sciences. We both had high organizational positions in EATCS, the European Association for Theoretical Computer Science.

I did not meet Ferenc after my visit to Szeged in August 2007, but we still corresponded very much after that. Sadly I followed his deteriorating health.

Dear Super-Brother Feri, I miss you deeply. So do my family and all your friends in Finland. 